\title{
Changes in the roles of transrectal ultrasonography for the diagnosis of prostate cancer
}

\author{
Atsushi Ochiai ${ }^{1}$
}

(C) The Japan Society of Ultrasonics in Medicine 2016

Transrectal ultrasonography (TRUS) of the prostate has been widely used for the diagnosis of prostate cancer since the first practical tomogram of the prostate was obtained in Japan half a century ago. The ability of conventional grayscale imaging was limited to differentiating malignant from benign lesions. The most common ultrasound appearance of prostate cancer is hypoechoic with approximately onethird of cancers being isoechoic. As serum prostate cancer antigen (PSA) is highly sensitive to prostate cancer detection, TRUS-guided systematic prostate biopsy (sampling tissues from designated areas irrespective of ultrasound findings) has been the gold standard method for the detection of prostate cancer.

One decade ago, we reviewed the prostate biopsy technique [1]. Modifications of TRUS-guided systematic biopsy were discussed. The number of cores and locations sampled were discussed with the aim of detecting more cancers. Extended biopsy approaches at lateral lesions decreased the false-negative rate, and an extended TRUSguided systematic biopsy strategy was recommended. Furthermore, biopsy findings such as cancer burden (number of positive cores and percent of cores positive for cancer) and Gleason score could preoperatively help predict the pathologic outcome at prostatectomy. In spite of these efforts, the strategies still caused us to miss clinically

Atsushi Ochiai

chikamika2001@yahoo.co.jp

1 Department of Urology, Aiseikai Yamashina Hospital, Kyoto, Japan significant cancer as well as to detect clinically insignificant cancer, leading to overdiagnosis and overtreatment of prostate cancer.

In recent years, multiparametric MRI (T2-WI, DWI, dynamic) dictated by the biological behavior of the underlying tumor has been successful at detecting prostate cancer. MRI-based biopsy has been shown to detect more clinically significant prostate cancer and less insignificant prostate cancer compared to systematic biopsy. Two approaches to TRUS-guided target biopsy based on MRIpositive lesions have been developed: cognitive fusion and MRI-TRUS fusion. Registration errors may occur with both TRUS-guided target approaches. Prostate biopsy performed under MRI guidance has also become available with an additional device and software. In the case of MRIguided in-bore biopsy, TRUS would no longer be utilized in the diagnostic process. However, the costly and complicated procedures would be unlikely to be popular in practice.

I reviewed the recent advances in transrectal ultrasonography of prostate cancer several years ago [2]. Moreover, we completed the clinical practice guidelines for ultrasound elastography in this journal [3]. The biological and functional characteristics of tumors such as the anatomical architecture, cellular density, and angiogenesis can be evaluated by ultrasound elastography and contrast ultrasonography. These new technologies can detect prostate cancer more efficiently than TRUS-guided systematic biopsy. Thus, similar to multiparametric MRI, a multiparametric US-based approach including B-mode, elastography, and dynamic contrast ultrasonography for detecting prostate cancer may be promising and should be established as a very accurate, widely available, cost-effective prostate biopsy in the near future. 


\section{References}

1. Ochiai A, Babaian R. Update on prostate biopsy technique. Curr Opin Urol. 2004;14:157-62.

2. Ochiai A. Update on transrectal ultrasonography of prostate cancer. Jpn J Med Ultrasonics. 2010;37:97-105.
3. Terminology and Diagnostic Criteria Committee. Japan Society of Ultrasonics in Medicine. Clinical practice guidelines for ultrasound elastography: prostate. J Med Ultrasonics. 2016;43:449-55. 